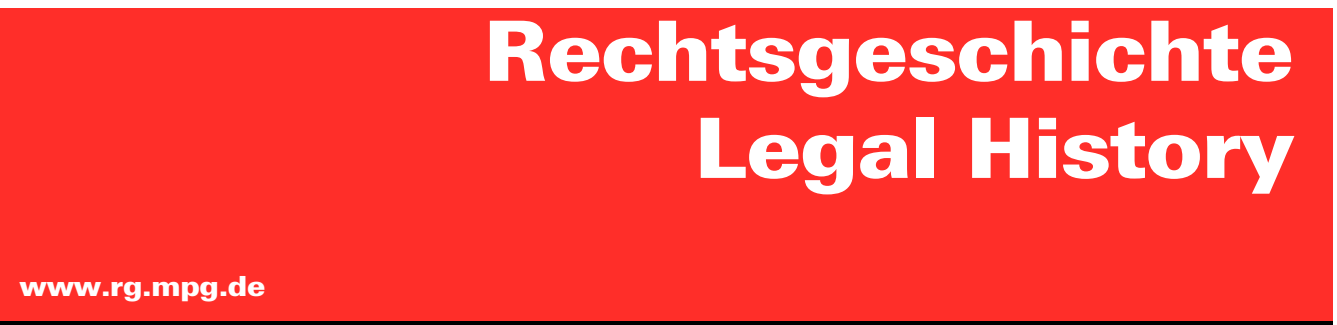

http://www.rg-rechtsgeschichte.de/rg26

$\operatorname{Rg} 26_{2018}$

$385-390$

Zitiervorschlag: Rechtsgeschichte - Legal History Rg 26 (2018)

http://dx.doi.org/10.12946/rg26/385-390

\title{
Wolfram Brandes*
}

\section{Jahre Papstgeschichte}

[1000 Years of Papal History]

* Akademie der Wissenschaften zu Göttingen / Goethe-Universität Frankfurt am Main / Max-Planck-Institut für europäische Rechtsgeschichte, Frankfurt am Main, brandes@rg.mpg.de

Dieser Beitrag steht unter einer Creative Commons cc-by-nc-nd 3.0

@creative $(1) \otimes \ominus$ 
chapters, if not all, succeed in mediating relevant and sometimes technically complex matters in a concise and understandable way. Also, more practical, methodological forms of knowledge (e.g. about data modelling, image digitisation, and legal situations) are conveyed in a manner that would serve anyone at the initial and planning stages of a research project with digital components. This applies especially from the perspective of (legal) history, where research based on quantitative data, for instance, has a long tradition, and the book covers »digital« topics that certainly may be relevant to (legal) historians. These include the introductions to digital data modelling, network theory and the creation of data collections as well as the excellent chapters on geographical information systems and information visualisation. Further, perhaps any research could be confronted - at certain points in the course of a digital research project in legal history - with many of the topics presented in this collection. And even those not directly participating in a digitally driven project will appreciate the book as a comprehensive "conversation course « to learn about relevant topics and terminology of the current (and not just the German-speaking) digital humanities.

\section{Wolfram Brandes}

\section{Jahre Papstgeschichte*}

Catharina Gowers, Waldemar Könighaus, Marcus Schütz, Cornelia Scherer, Thorsten Schlauwitz, Victoria Trenkle, Judith Werner und natürlich dem spiritus rector des Unternehmens und einem der besten Kenner der Papstgeschichte, Klaus Herbers, kann man nur den größten Dank aussprechen, dass sie sich der höchst mühsamen und komplizierten Aufgabe angenommen haben, den »Jaffé« in einer dritten Auflage zu überarbeiten.

Das tragische Schicksal des Philipp Jaffé (18191870) kann hier nicht thematisiert werden, wirft aber ein bezeichnendes Licht auf die deutsche Me- diävistenszene im 19. Jahrhundert. Erinnert sei an seine sehr unschönen Auseinandersetzungen mit Georg Heinrich Pertz und den Monumenta Germaniae Historica, die Publikation seiner Bibliotheca rerum Germanicarum I-VI (1864-1873) - durchaus als Konkurrenz zu den MGH gedacht - und seinen tragischen (bis heute nicht wirklich geklärten) Selbstmord. ${ }^{1}$ In seinem 32 . Lebensjahr (!) erschienen die Regesta pontificum Romanorum (1851), die dann am Ende des Jahrhunderts unter der Leitung von Wilhelm Wattenbach von Samuel Löwenfeld, Ferdinand Kaltenbrunner und Paul Ewald in einer
Regesta pontificum Romanorum ab condita ecclesia ad annum post Christum natum MCXCVIII edidit Phillipus Jaffé, editionem tertiam emendatam et auctam iubente Academia Gottingensi sub auspiciis Nicolai Herbers, tomus primus [sic!] (a S. Petro usque ad a. DCIV) curavit Marcus Schütz cooperantibus Victoria TrenkLe, IUdith WerNER itemque Catharina Gowers, Waldemaro Könighaus, Cornelia Scherer, Thorstano Schlauwitz, Gottingae (in aedibus Vandenhoeck et Ruprecht) MMXVI, XV et 584 p.; ISBN 978-3-525-31003-8;

Regesta pontificum Romanorum ab condita ecclesia ad annum post Christum natum MCXCVIII edidit Phillipus Jaffé, editionis tertiae emendatae et auctae iubente Academia Gottingensi sub auspiciis NicoLaI Herbers tomum secundum (ab a. DCIV ad a. DCCCXLIV) curaverunt Waldemarus KöNIGHaUs, ThorStanus SCHLaUwITZ cooperantibus Cornelia Scherer, Marco Schütz, Gottingae (in aedibus Vandenhoeck et Ruprecht) MMXVII, XVI et 406 p., ISBN 978-3-525-31035-9; Regesta pontificum Romanorum ab condita ecclesia ad annum post Christum natum MCXCVIII edidit Phillipus Jaffé, editionis tertiae emendatae et auctae iubente Academia Gottingensi sub auspiciis Nicolai Herbers, tomum tertium (ab a. DCCCXLIV usque ad a. MXXIV) curavit IUDITH WeRNER cooperante Waldemaro Könighaus, Gottingae (in aedibus Vandenhoeck et Ruprecht) MMXVII, XV et 708 p., ISBN 978-3-525-31036-6

1 Schwartz (2016) (Rezension von K. Herbers in: https://www.hsoz kult.de/publicationreview/id/rez buecher-27485); siehe auch ScHWARTZ (2015); immer noch wichtig ist BRESSLAU (1921) bes. $326 \mathrm{ff}$. und passim. 
stark überarbeiteten zweiten Auflage à jour gebracht wurden (Leipzig 1885/1888).

Natürlich mussten sich die Bearbeiter der dritten Auflage bei der Einarbeitung (oder wenigstens Notierung) der zu zitierenden (relevanten) Sekundärliteratur sehr beschränken. Dass sie sich dabei besonders auf die seit 1888 erschienenen Regestenwerke $^{2}$ konzentrierten, lag in der Natur der Sache. Sicher findet jeder Benutzer mindestens ein Regest (in der Regel wohl mehrere oder sogar sehr viele), in dem er diese oder jene gelehrte Abhandlung vermisst. Es ist ja davon auszugehen, dass es heute wohl niemanden mehr gibt, der die Papstgeschichte von Petrus bis zu Innozenz III. wirklich überblickt, also mehr als 1200 Jahre intensivst erforschter Geschichte. Dies trifft auch auf den Rezensenten zu, der sich entsprechend auf die Zeiten konzentriert, mit denen er sich eingehender beschäftigt hat.

Die im ersten Band an zahlreichen Stellen zitierten Regesten von Otto Seeck aus dem Jahre $1919^{3}$ hätte man sich an (sehr) vielen Stellen sparen können, sind sie doch heute restlos überholt. Immerhin hätte man vom Untertitel dieses Nachschlagewerkes (»Vorarbeit zu einer Prosopographie der christlichen Kaiserzeit «) ausgehend die inzwischen für die Spätantike und das frühe Mittelalter erschienenen prosopographischen Referenzwerke nutzen können. ${ }^{4}$

$\mathrm{J}^{3}+380$ betrifft das berühmte Constitutum Constantini. Hier werden - in aller gebotenen Kürze (aber hinreichend ausführlich) - die divergierenden Datierungen (vor 800 gemäß dem main stream und nach 800 - Johannes Fried [und der Rezensent]) angesprochen und die nötigen bibliographischen Angaben geboten. Nicht berücksichtigen konnten die Autoren den 2015 erschienenen Aufsatz von Federico Montinaro, ${ }^{5}$ der zeigt, dass die Passagen im Liber pontificalis, die insbesondere den

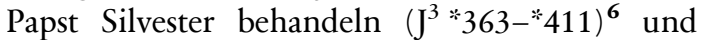
Schenkungen Konstantins des Großen an die römische Kirche erwähnen, viel spätere »Fälschungen« darstellen und ahistorisch sind. Die Anzahl der mit einer crux versehenen Regestennummern müsste sich danach erheblich vermehren! Gerade dieses Beispiel demonstriert anschaulich, vor welchen Problemen man bei der Arbeit an Regesten steht. Die Forschung schreitet schnell voran (sehr viel Unsinn, den es zu erkennen gilt, wird ebenfalls permanent produziert) und man kann bei einem derartigen Großprojekt nicht bei allen Details den aktuellen Forschungsstand präsentieren. Auch der inzwischen erschienene dritte Band der monumentalen Edition der Akten des Nicaenum II von Erich Lamberz konnte (natürlich) nicht (mehr) benutzt werden, was schade, aber nicht zu ändern ist. ${ }^{7}$

Einen großen Teil des ersten Bandes nehmen naturgemäß die Regesten zum Pontifikat Gregors (I.) des Großen ein, dessen Register ja überliefert wurde (348-509; J ${ }^{3}$ *2071-*3164). Hier konnten die Bearbeiter umfassend auf die Italia pontificia zurückgreifen. Nützlich wären Hinweise auf die entsprechenden Bände der Prosopographie chrétienne oder der Prosopopgraphy of the Later Roman Empire (bei den weltlichen Empfängern - z. B. $\mathrm{J}^{3}$ 2148, 2206, 2207, 2209, 2311, 2315 usw.) sowie der Prosopographie der mittelbyzantinischen Zeit gewesen. ${ }^{\mathbf{8}}$ In diesen Werken werden die Empfänger von Papstbriefen behandelt. Auch die (gefälschten) Papstbriefe des Pseudo-Isidor finden sich - stets mit crux. Überhaupt (und das gilt natürlich für alle Bände) stellt diese neue Auflage des »Jaffé« eine wesentliche Grundlage jeder kanonistischen Forschung (und darüber hinaus) dar, ist also ein nicht zu ignorierendes Hilfsmittel für die mittelalterliche Rechtsgeschichte bis zum ausgehenden 12. Jahrhundert.

Der zweite Band umfasst die Zeit von Sabinianus (604-606) bis Gregor IV. (827-844). Man sehe es dem Rezensenten nach, dass er sich diesem Band besonders zuwendet, betrifft er doch einige bevorzugte Forschungsgegenstände desselben.

Hatte die zweite Auflage (220) "nur« zwei nichtssagende Einträge zu Papst Sabinianus, dem Nachfolger Gregors des Großen, kann man jetzt 7 Regesten $\left(\mathrm{J}^{3} * 3165-* 3171\right)$ finden. Darunter ein damals übersehener Brief (dessen Datierung allerdings umstritten ist) des Columban von Luxeuil $\left(\mathrm{J}^{3}\right.$ 3166). Ähnlich verhält es sich mit den folgenden Päpsten.

\footnotetext{
2 Siehe u. a. Dölger / Müller (2009); Dölger / MÜller (2003); DölgeR/ WirTh (1995); GRUMEL (1932); Grumel/ Darrouzès (1989); LOUNGHIS / Blysidou / LAMPAKES (2005).
}

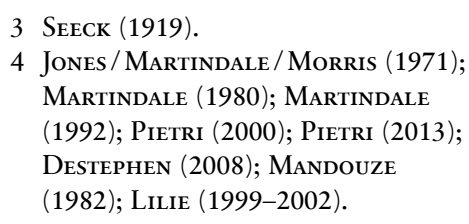

\author{
5 Montinaro (2015). \\ 6 Vgl. Liber Pontificalis (Duchesne \\ 1955) 170-187. \\ 7 Nicaenum II (LAmberz 2016). \\ 8 Siehe oben Anm. 4 und 5.
}


Durch die Benutzung der Regesten (z. B.) von Conte, den Regesten der Kaiserurkunden des oströmischen Reiches, ${ }^{9}$ der Gallia pontificia, Germania pontificia, Italia pontificia, Iberia pontificia usw., der neuen Bände der Regesta Imperii ${ }^{\mathbf{1 0}}$ - neben anderen Werken, die nach der zweiten Auflage erschienen ${ }^{11}$ - wurde auch in diesem Band die Anzahl der Regesten ganz erheblich vermehrt. Ob das immer sinnvoll ist, sei dahingestellt. Ein Beispiel (das genügen soll, denn es gibt viele derartige Fälle): Warum wurde das Ewald-Jaffésche Regest *1995 in J *3173 und *3174 aufgespalten? Sehr oft folgen die Verfasser Conte, der ohnehin eine (bedenkliche?) Tendenz zur "Vermehrung" von Regesten aufwies, ohne dass sich der Sinn schnell erschließt (sofern einer vorhanden war bzw. ist). Zwar ist klar, dass zu jedem Regest nicht die aktuelle Literatur aufgelistet werden kann (ansonsten käme das Projekt nie zu einem Ende), aber gelegentlich hätte doch auf neuere Literatur Bezug genommen werden sollen, z. B. in $\mathrm{J}^{3}$ ?3327 und ?3328 auf eine erst vor wenigen Jahren erstmals publizierte Konzilssynopse (als weitere Quelle) und auf einige grundlegende Werke. ${ }^{12}$

Es gibt allerdings bisweilen Fälle, bei denen es nicht auf den ersten Blick klar ist, ob eine Aufnahme ins Regestenwerk angebracht ist, oder eben nicht. Erich Caspar, ${ }^{13}$ dessen monumentale Papstgeschichte den Verfassern der dritten Auflage selbstverständlich bekannt ist, wie diverse Verweise zeigen, notierte in seiner Behandlung eines Konflikts zwischen Papst Benedikt II. und Julian von
Toledo lapidar: »Nicht in Jaffés Regesten verzeichnet. ${ }^{14}$ Der Hintergrund ist einfach berichtet (und war schon mehrfach Gegenstand wissenschaftlicher Untersuchungen ${ }^{15}$ ): Papst Leo II. (681-683) hatte die Akten des Constantinopolitanum III, des sechsten ökumenischen Konzils (681/682), das dem monenergetisch-monotheletischen Streit ein Ende setzte, ${ }^{16}$ teilweise (Horos, prosphonetische Akklamationen und das kaiserliche Edikt ${ }^{17}$ ) übersetzen lassen und zusammen mit vier Briefen (JE 2119-2122 $=\mathrm{J}^{3}$ 3504-3507) durch den notarius regionarius Petrus nach Spanien gesandt. ${ }^{18}$ Hier war gerade das XIII. Konzil von Toledo zu Ende gegangen und die Bischöfe befanden sich auf dem Rückweg in ihre Diözesen. Deshalb sandte man ihnen Kopien der aus Rom eingetroffenen Auszüge aus den Akten des Constantinopolitanum III (in lateinischer Übersetzung natürlich) zu, damit sie zustimmen konnten. Diese zustimmenden Schriftstücke sandte man wieder zurück nach Rom. Erzbischof Julian von Toledo fügte dem noch einen eigenen theologischen Text, das sog. Apologeticum (Text verloren), bei. Noch vor der Einberufung des XIV. Konzils von Toledo (14.11.684) ${ }^{19}$ ging eine hispanische Gesandtschaft zusammen mit dem erwähnten notarius regionarius Petrus nach Rom ab, um diese Schriftstücke dem Papst zu überreichen. ${ }^{20}$ Inzwischen war Leo II. gestorben und Benedikt II. war nun im Amt. Dieser kritisierte den Inhalt des Apologeticum des Julian von Toledo, offenbar mündlich gegenüber den spanischen Gesandten, ${ }^{21}$ die die päpstlichen Ein-
9 Conte (1971); Conte (1984); nicht benutzt wurde (leider) Cosentino (1996) und Cosentino (2000).

10 BÖHMER/MüHLbacher (1908).

11 Für den sog. Monotheletismus siehe bes. WinkelmanN (2001).

12 Konzilssynopse (2013) 168 f.; erstaunlich ist der Umstand, dass das einschlägige Werk von ScHoLz (2006) nicht benutzt wurde. Das ist ein Manko! Und wieso zu diversen Regesten im Umfeld des Nicaenum II ausgerechnet CHIFAR (1993) (z. B. $\mathrm{J}^{3} 4494$ u. a.) zitiert wird, statt das unübertroffene Standardwerk zu allen Fragen des sog. Bilderstreits Brubaker/Haldon (2011), wo auch alle Papstbriefe in den Osten ab Gregor II. ausführlich und kritisch behandelt werden, zu benutzen, erstaunt den Rezensenten.
13 Caspar (1933) bes. $614 \mathrm{ff}$. zu Benedikt II.

14 Caspar (1933) 674 Anm. 2.

15 ORLANDis / Ramos-Lissón (1981) 272-293; Strohm (1983); Riedinger (1988) (auch in: RiEdinger [1998] 261-280); Julian of Toledo, Prognosticum (trad. STANCATI) bes. 132-155 (mit der älteren Literatur).

16 Constantinopolitanum III (RIEDINGER [1990/1992]); vgl. WINKELMANN (2001), Nr. 161.

17 Winkelmann (2001), Nr. 165; DÖLGER/Müller (2003), Nr. 247.

18 Martinez Diez/Rodriguez (1982), 181-205 (190-204); WinkELMANN (2001), Nr. 168a.

19 Orlandis / Ramos-Lissón (1981) 276; WinkelmanN (2001), Nr. 168b.

20 Vives (1963) 453 = Julianus Toletanus (Hillgarth 1976) 129.
21 Ebenda: Post huius igitur piae confessionis prolatam devotis vocibus regulam ad illa nos illico convertimus contuenda capitula, pro quibus muniendis ante hoc biennium beatae memoriae Romanus papa Benedictus nos litterarum suarum significatione monuerat, quae tamen non in scriptis suis annotare curavit sed homini nostro verbo renotanda iniunxit, ad quod illi iam eodem anno sufficienter congruesque responsum est, nos tamen nunc eandem renotationem hominis nostri studiosius relegentes invenimus quod in libro illo pesponsionis fidei nostrae, quem per Petrum regionarium Romanae ecclesiae miseramus, ... Z $\mathrm{Z}$ den theologischen Einwänden des Papstes siehe OrLandis / RamosLissón (1981) 284-286. 
wände aufschrieben und nach Spanien brachten. Sollte man solch einen Vorgang in einem Regest notieren? Ich glaube, ja; doch mag man es auch ablehnen. Caspar jedenfalls monierte das Fehlen eines Regests. ${ }^{\mathbf{2 2}}$ Und auch die historiographische Überlieferung (Continuatio Hispana der Geschichte des Isidors von Sevilla bzw. die sog. mozarabische Chronik) legt ein Regest nahe. ${ }^{23}$ Der Papst habe das Buch indiziert (indixerat). Auf dem XV. Konzil von Toledo im Frühjahr 688 (ab 11. Mai) ${ }^{\mathbf{2 4}}$ wurde die Angelegenheit behandelt, und dem Papst wurde »Oberflächlichkeit « vorgeworfen. Julian hatte inzwischen sein Apologeticum de tribus capitulis (CPL 1259) geschrieben (686), welches das Konzil feierte. Es wurde nach Rom gesandt, wo inzwischen Papst Sergius I. amtierte. Dieser schätzte die Ansichten Julians als iusta et pia ein und der Stolz des westgotischen Episkopats war wiederhergestellt. Dies besagt auch J3 3556* (Sergius I.) - ein »neues« Regest und der Vorgang wurde tatsächlich von Jaffé und Ewald (bzw. Wattenbach) übersehen. Ich habe diese interessanten Vorgänge hier etwas ausführlicher dargestellt, als sonst in einer Rezension üblich, um zu zeigen, wie kompliziert im Detail die Arbeit an dieser dritten Auflage gewesen sein muss. Wollte man stets jedes einzelne Regest bzw. seine Entstehungsumstände in aller Ausführlichkeit analysieren, hätte das Unternehmen noch viele Jahrzehnte gedauert - und niemandem hätte das genutzt.

Der dritte Band profitiert in erheblichem Maße von Herbers' Vorarbeiten. Zu nennen sind seine monumentale Monographie über Leo IV. ${ }^{25}$ sowie zwei von den im Rahmen der Regesta imperii erschienenen Bände der Papstregesten zum 9. Jahrhundert, die er selbst beisteuerte. ${ }^{26}$ Der Band reicht bis Benedikt VIII. (1012-1024) und ist naturgemäß der bisher umfangreichste, wird aber ver- mutlich vom dem noch ausstehenden vierten Band übertroffen werden.

Manchmal kann man sich des Eindrucks nicht erwehren, dass etwas zu viel des Guten getan wurde. Z. B. entnahmen die Autoren Geertmanns "More veterum ${ }^{27}$ eine Unzahl neuer Regesten (es dürften einige Hundert sein). Ob das wirklich notwendig war, wagt der Rezensent zu bezweifeln.

Dass das gesamte Werk auf Latein (einschließlich der Vorworte; am Ende der Bände findet man schließlich auch eine deutsche Fassung) verfasst ist, erscheint heute etwas skurril. Und vermutlich wird dieser Umstand nicht gerade zu einer häufigeren Benutzung beitragen, was natürlich auch Herbers und seine Crew wussten. Aber dieser Umstand ergab sich wohl aus praktischen Erwägung, denn die ersten beiden Auflagen waren (anders als die Böhmer-Mühlbacherschen Regesta imperii z. B.) auf Latein. Und schon Jaffé pflegte einen ausgesprochen elaborierten lateinischen Stil. Die dritte Auflage übernahm die lateinischen Regestentexte der Vorgänger, was zweifellos viel Zeit sparte. Insofern ist jede Kritik fehl am Platz!

Leider ist man nunmehr gezwungen, gewohnte Regestennummern zu korrigieren. Diese Mühe wird durch die dem dritten Band beigegebenen Konkordanzen (etwas) erleichtert, wobei diese großformatigen Bände schnell den halben Schreibtisch blockieren!

Abschließend bleibt nur noch die angenehme Pflicht, Klaus Herbers und seinen Mitarbeitern nochmals einen großen Dank abzustatten. Der neue Jaffé gehört in jede einschlägige Bibliothek und in den Handapparat jedes Gelehrten, der im ersten Millennium unterwegs ist. Dagegen steht nur der stolze Preis von 500 Euro für diese drei Bände.
22 Siehe eben bei Anm. 14.

23 Chron. Mozarab. 754 (1980) $60=$ Continuatio Hispana (1894) 350; vgl. Wolf (1990) $127 \mathrm{f}$.

24 Orlandis / Ramos-Lissón (1981) bes. 277-293 zum Toletanum XV; Vives (1963) 453-456 (Behandlung des
Julianischen Apologeticum auf dem Toletanum XV).

25 Herbers (1996).

26 BÖHMER/HERBERs (1999); BÖHMER/

Herbers (2012) und ein weiterer

Band (für 867-872 [Hadrian II.])

wird gegenwärtig vorbereitet. Über- blick über die relevanten Bände der

Regesta imperii im dritten Band von $\mathrm{J}^{3}$ S. $663 \mathrm{f}$.

27 Geertmann (1975). 
Bibliographie

\section{Quellen}

- Constantinopolitanum III (RIedinger [1990/1992]) = Concilium universale Constantinopolitanum tertium, ed. Rudolf Riedinger (Acta conciliorum oecumenicorum, ser. II, II/1-2), Berlin

- Chron. Mozarab. 754 (1980) = Crónica Mozarabe de 754. Edición crítica y traducción, por José Eduardo López Pereira (Textos medievales 58), Zaragoza

- Continuatio Hispana (1894), Continuationes Isidorianae, continuatio Hispana, ed. Theodor Mommsen, MGH Auctores antiquissimi XI (Chronica minora II), Berlin

- Julian of Toledo, Prognosticum (trad. Stancati) (2010) = Julian of Toledo, Prognosticum futuri saeculi, transl., ed., and introd. by Tommaso Stancati (Ancient Christian writers 63), New York

- Julianus Toletanus (Hillgarth 1976) = Sancti Ivliani Toletanae sedis espiscopi opera, pars 1, ed. Jocelyn N. Hillgarth (Corpus christianorum, Series Latina 115), Turnhout

- Konzilssynopse (2013) = Eine unbekannte Konzilssynopse aus dem Ende des 9. Jahrhunderts, ediert, übersetzt und kommentiert von Lars Hoffmann und Wolfram Brandes (Forschungen zur byzantinischen Rechtsgeschichte 30), Frankfurt am Main

- Liber Pontificalis (Duchesne 1955) = Le Liber Pontificalis. Texte, introduction et commentaire par Louis DuchesNe, Paris (Nachdr.)

- Martinez Diez, Rodriguez (1982) = Martinez Diez, Gonzalo, Félix Rodriguez, La colección canónica Hispania, III: Concilios griegos y Africanos (Monumenta Hispaniae sacra, Serie Canónica III), Madrid

- Nicaenum II (LAmberz 2016) = Consilium universale Nicaenum secundum, concilii actiones VI-VII, ed. Erich Lamberz (Acta conciliorum oecumenicorum, ser. II, III/3), Berlin/Boston

- Vives (1963) = Concilios Visigóticos e Hispano-Romanos. Edición preparada por José Vives, Barcelona / Madrid

- Wolf $(1990)=$ Conquerors and Chronicles of Early Medieval Spain. Translated with notes and introduction by KenNeth B. Wolf (Translated Texts for Historians 9), Liverpool

\section{Literatur}

- Böhmer, Johann F., Engelbert Mühlbacher (1908), Regesta imperii, I: Die Regesten des Kaiserreichs unter den Karolingern 751-918, nach Johann F. Böhmer neubearbeitet von Engelbert Mühlbacher, Innsbruck (Nachdr. Hildesheim 1966)

- Böhmer, Johann F., Klaus Herbers (1999), Regesta Imperii I. Die Regesten des Kaiserreiches unter den Karolingern 751-918 (926/962), vol. 4: Papstregesten 800-911, Teil 2: 844-872, Lfg. 1: 844-858, bearb. von KLaus Herbers, Köln/Weimar/Wien

- Böhmer, Johann F., Klaus Herbers (2012), Regesta imperii I. Die Regesten des Kaiserreiches unter den Karolingern 751-918 (987), vol. 4: Papstregesten 800-911, Teil 2: 844-872; Lfg. 2: 858-867 (Nikolaus I.), bearb. von KLaus Herbers, Wien/Köln/ Weimar

- Bresslau, Harry (1921), Geschichte der Monumenta Germaniae Historica, Hannover

- Brubaker, Leslie, John Haldon (2011), Byzantium in the Iconoclast Era, c. 680-850: a history, Cambridge

- Caspar, Erich (1933), Geschichte des Papsttums, II: Das Papsttum unter byzantinischer Herrschaft, Tübingen

- Chifar, Nicolae (1993), Das VII. Ökumenische Konzil von Nikaia: Das letzte Konzil der ungeteilten Kirche (Oikonomia 32), Erlangen

- Conte, Pietro (1971), Chiesa e primato nelle lettere dei papi del secolo VII, Milano

- Conte, Pietro (1984), Regesto delle lettere dei papi del secolo VIII, Milano

- Cosentino, Salvatore (1996), Prosopografia dell'Italia bizantina (493-804), I, Bologna

- Cosentino, Salvatore (2000), Prosopografia dell'Italia bizantina (493-804), II, Bologna

- Destephen, Sylvain (2008), Prosopographie du diocèse d'Asie (325-641), Paris

- Dölger, Franz, Andreas E. Müller (2003), Regesten der Kaiserurkunden des Oströmischen Reiches von 565-1453, bearbeitet von Franz Dölger, 1. Teil, 1. Halbband: Regesten 565-867, 2. Aufl., unter Mitarbeit von Johannes Preiser-Kapeller und Alexander Riehle besorgt von Andreas E. Müller, München

- Dölger, Franz, Andreas E. Müller (2009), Regesten der Kaiserurkunden des Oströmischen Reiches von 565-1453, bearbeitet von Franz Dölger, 1. Teil, 2. Halbband: Regesten von 867-1025, 2. Aufl., bearbeitet von Andreas E. Müller unter verantwortlicher Mitarbeit von Alexander D. Beihammer, München

- Dölger, Franz, Peter Wirth (1995), Regesten der Kaiserurkunden des Oströmischen Reiches von 565-1453, bearbeitet von Franz Dölger, 2. Teil: Regesten von 1025-1204, 2., bearbeitete und verbesserte Aufl., bearbeitet von Peter Wirth, München

- Geertmann, Hermann (1975), More veterum. Il Liber pontificalis e gli edifici ecclesiastici di Roma nella tarda antichità e nell'alto medioevo (Archeologica Traiectina 10), Groningen

- Grumel, Venance (1932), Les regestes des actes du patriarcat de Constantinople, I/fasc. 1: Les regestes de 381 à 715, Kadiköy (Nachdr. Paris 1972)

- Grumel, Venance, Jean Darrouzès (1989), Les regestes des actes du patriarcat de Constantinople, I/fasc. 1 et 2, Paris

- Herbers, Klaus (1996), Leo IV. und das Papsttum in der Mitte des 9. Jahrhunderts. Möglichkeiten und Grenzen päpstlicher Herrschaft in der späten Karolingerzeit (Päpste und Papsttum 27), Stuttgart

- Jones, Arnold H.M., John R. Martindale, John Morris (1971), The Prosopography of the Later Roman Empire I (A. D. 260-395), Cambridge

- Lilie, Ralph-Johannes, Claudia Ludwig, Thomas Pratsch, Ilse Rochow (1999/2000/2001/2002) = Prosopographie der mittelbyzantinischen Zeit. Erste Abteilung (641-867), I-VI. Nach Vorarbeiten von Friedhelm WinkelmanN, unter Mitarbeit von Wolfram Brandes, John R. Martindale und Beate Zielke, Berlin/New York 
- Lounghis, Telemachos C., Basilike Blysidou, Stelios Lampakes (2005), Regesten der Kaiserurkunden des oströmischen Reiches von 476 bis 565 , Nicosia

- Mandouze, André (1982), Prosopographie de l'Afrique chrétienne (303-533), Paris

- Martindale, John R. (1980), The Prosopography of the Later Roman Empire II (A. D. 395-527), Cambridge

- Martindale, John R. (1992), The Prosopography of the Later Roman Empire III (A. D. 527-641), Cambridge

- Montinaro, Federico (2015), Les fausses donations de Constantin dans le Liber pontificalis, in: Millennium 12, 203-229

- Orlandis, José D., Domingo Ramos-Lissón (1981), Die Synoden auf der Iberischen Halbinsel bis zum Einbruch des Islam (711), Paderborn u. a.

- Pietri, Charles, Pietri, Luce (2000), Prosopographie chrétienne du Bas-Empire, II: Prosopographie de l'Italie chrétienne (313-604), I-II, Rom

- Pietri, Luce (2013), Prosopographie de la Gaule chrétiennne (314-614), I-II, Paris

- Riedinger, Rudolf (1988), Die Dokumente des Petrus notarius regionarius auf seiner Reise von Rom nach Spanien im Jahre $683 / 4$, in: Burgense 29, 233-250

- Riedinger, Rudolf (1998), Kleine Schriften zu den Konzilsakten des 7. Jahrhunderts (Instrumenta Patristica 34), Steenbrugge / Turnhout

- Scholz, Sebastian (2006), Politik - Selbstverständnis - Selbstdarstellung. Die Päpste in karolingischer und ottonischer Zeit (Historische Forschungen 26), Stuttgart

- Schwartz, Daniel R. (2015), New Light and Remaining Questions Concerning the Death of Philipp Jaffé, in: Concilium Medii Aevi 8, 61-80

- Schwartz, Daniel R. (2016), Between Jewish Posen and Scholarly Berlin. The Life and Letters of Philipp Jaffé, Berlin

- Seeck, Отto (1919), Regesten der Kaiser und Päpste für die Jahre 311 bis 476 n. Chr. Vorarbeit zu einer Prosopographie der christlichen Kaiserzeit, Stuttgart (Nachdr. Frankfurt am Main 1964)

- Strohm, Martin (1983), Der Konflikt zwischen Erzbischof Julian von Toledo und Papst Benedikt II.: Ein Faktum von ökumenischer Bedeutung, in: Annuarium historiae conciliorum 15, 249-259

- Winkelmann, Friedhelm (2001), Der monenergetisch-monotheletische Streit (Berliner Byzantinistische Studien 6), Frankfurt am Main u. a.

\section{Caspar Ehlers}

\section{Integration durch Recht*}

In seinem neuen Buch bietet Karl Ubl einen weit gefassten Überblick seiner bisherigen Forschungen zur Lex Salica im Kontext seines Projektes an der Universität Köln zu den frühmittelalterlichen Rechtsüberlieferungen, beginnend mit den substantiellen Beobachtungen »Warum Barbaren Gesetze erlassen « (37-66) als Folge aus der Frage nach Einsatz und Nutzen der Rechtsbücher (Einleitung, 11-35). Er sieht die fränkische Lex in ihren Ursprüngen als identitätsstiftendes Instrument an (»Ein Monument der Alterität«, 67-97, sowie als "Entwürfe von Gemeinschaft im 6. Jahrhundert", 99-135).

Die »Neufassung Pippins I.« bedeutete einen aus heutiger Sicht erkennbaren Eintritt in die schriftliche Überlieferung (137-163), die Neufas- sung Karls des Großen sogar eine Mystifizierung der Autorität des Rechts (165-191) vor der transformatorischen Phase des Untergangs des fränkischen Rechts (193-219) im Jahrhundert der Nachfolger Karls des Großen (zusammenfassend: „von der Lex Salica zu den Leges Francorum «, 218 f.). Das »Wissen über das Recht der Franken im 9. Jahrhundert « ist Gegenstand des achten Kapitels (221244).

Ausgehend von der Beobachtung, dass die älteste Fassung der Lex Salica wegen ihres archaischen Charakters kaum geeignet erscheint, das Rechtsbuch eines zur Großmacht aufsteigenden Reiches zu werden (11 ff.), referiert Ubl die Forschungskontroversen um die Deutung der Lex als »Urtext der deutschen Rechtsgeschichte« (13-24) und um 